\title{
THREE-POINT BENDING OF AN EXPANDED-TAPERED BEAM WITH CONSIDERATION OF THE SHEAR EFFECT
}

\author{
Krzysztof MagnuCKi, JeRzy LeWinski \\ Eukasiewicz Research Network - Institute of Rail Vehicles "TABOR", Poznan, Poland \\ e-mail: krzysztof.magnucki@tabor.com.pl \\ Ewa Magnucka-Blandzi \\ Poznan University of Technology, Institute Mathematics, Poznan, Poland \\ Hanna STAWECKA \\ Lukasiewicz Research Network - Institute of Rail Vehicles "TABOR", Poznan, Poland
}

\begin{abstract}
The paper is devoted to an expanded-tapered beam of rectangular cross section subjected to three-point bending. The analytical model of the beam is formulated with consideration of a non-linear hypothesis of the cross section deformation. The problem of shear stress distribution in the beam is analysed based on the above mentioned hypothesis. Moreover, a numerical FEM model (SolidWorks) is developed. Examplary computations have been carried out based on the analytical and numerical models.
\end{abstract}

Keywords: expanded-tapered beam, shear deformation theory, shear stresses

\section{Introduction}

The Euler-Bernoulli beam theory is formulated based on a linear hypothesis. The classical theory of beams does not take into account the shear effect. Timoshenko (1921) initiated consideration of the shear effect in beam bending. Wang et al. (2000) described in details the shear effect problems in the case of bending of beams and plates. They presented, in particular, the Euler-Bernoulli, Timoshenko and Reddy-Bickford beam theories. These cases were illustrated for various variants of boundary conditions. The expanded-tapered beam problems with consideration of stress, displacements and force relationships were also presented. Zhou and Cheung (2001) investigated free vibrations of various tapered Timoshenko beams. The eigenfrequency equation of the beams was formulated with the help of the Rayleigh-Ritz method. The approach ensured good compliance of the results with those ones presented in the literature. Auciello and Ercolano (2004) proposed a method of analysing Timoshenko beams with consideration of shear deformation. The iterative variational Rayleigh-Ritz method was applied to solve the problem, as an alternative to usual FEM methods. Some numerical examples were developed and the results were compared to the ones obtained by other researchers. Maalek (2004) considered shear deflection of tapering cantilever beams of rectangular cross section. The problem was solved by integration of the Timoshenko beam equation and with the method of virtual work. The results well agreed with the data calculated with FEM and obtained in experiments. Dado and Al-Sadder (2005) focused on the problem of large deflections of prismatic and non-prismatic cantilever beams under various types of load. The angle of beam rotation was described by a polynomial, the coefficients of which were calculated from minimization of the integral of the residual error and boundary conditions. The results were compared to data computed with the MSC/NASTRAN computer package. Slivker (2007) described variational methods in application to theoretical modelling of the structures. The author presented examples of detailed solutions 
of beams of varying cross section with consideration of the shear effect. Rajasekaran (2008) used methods of differential quadrature and harmonic differential quadrature to solve the buckling problem of non-prismatic beams and columns. The governing differential equation was converted to algebraic ones with the use of differential transformation methods. The results were compared to those obtained from other numerical and analytical methods. De Rosa and Lippiello (2009) calculated free vibrations frequencies of tapered Euler-Bernoulli beams subjected to various constraints. The authors used the cell discretization method that allows one to determine stiffness and mass matrices, enabling one to solve the eigenvalue problem. Comparison to other results demonstrated good quality of the approach. Carrera and Giunta (2010) proposed some theories intended for analysis of beams. The analyses were carried out for various values of the span-to-height ratio. Comparison of the results with the solutions obtained by other authors confirmed their good agreement. Reddy (2010) used constitutive relations of Eringen and von Karman nonlinear strains with a view to develop new versions of beam and plate theories, with consideration of the shear effect. This theoretical approach enabled one to reach finite element results and to define influence of geometric nonlinearity on bending of beams and plates. Attarnejad et al. (2011) dealt with analysis of arbitrarily tapered Timoshenko beams using Basic Displacement Functions. This allowed one to derive the exact shape functions that, in turn, enabled one to interpolate shape functions in the finite element area. Numerical examples confirmed good efficiency of the method. Shahba et al. (2011) analyzed stability and free vibration of functionally graded tapered Timoshenko beams with the finite element method. The authors formulated a special finite element using exact shape functions with consideration of exact variations of the cross sectional profile. The numerical examples verified effectiveness of the method displaying the effects of the taper ratio and material non-homogeneity on the critical load and natural frequencies of the beams. Wang (2012) was concerned with deformation of a tapered cantilever beam. The author formulated explicit stability criteria of pointy tapered columns and presented numerical calculations related to flat-ended columns. The formulae for large deformation were derived and numerically integrated. It was shown that the deformation depended on the taper ratio and the cross section profile of the beam. Rajasekaran (2013) used the Differential Transformation Method and Differential Quadrature Element Method with a view to solve the problem of free vibration of functionally graded tapered Timoshenko beams. Effectiveness of the approach was assessed with consideration of the effect of shear deformation, material non-homogeneity and the taper angle. The results were compared to those previously obtained by other authors. Huang et al. (2013) presented a simple higher-order theory intended for analysis of natural vibration of beams of circular cross section. The new model was close to the Levinson theory of rectangular beams. The natural frequencies calculated that way were compared with success to those obtained with the use of the Timoshenko and Euler-Bernoulli beam theories and with the Finite Element Method. Taha and Nassar (2014) studied axially-loaded tapered beams supported on an elastic two-parametric foundation. The authors formulated classical equations of motion without consideration of the shear effect in the beam. The analytical solutions were compatible with results obtained from other techniques. Auricchio et al. (2015) dealt with a beam having a cross section varying along the beam axis. A corresponding finite element was developed. The authors adopted the Hellinger-Reissner functional meeting the equilibrium equations. The results demonstrated that the beam model and the developed finite element properly predicted distributions of displacements and stress in tapered and arch-shaped beams. Balduzzi et al. (2016) investigated simple compatibility, equilibrium and constitutive equations of a nonprismatic planar beam. A model was developed that accurately depicted the impact of the beam non-prismatic geometry. A number of analytical and numerical results was compared to those available in the literature. It was found that the proposed model sufficiently accurately predicted the real behaviour of non-prismatic beams. Trahair and Ansourian (2016) dealt with tapered web I-beams and adopted radial instead of parallel normal stress trajectories. As a result, the shear 
stress distribution is affected by vertical components of inclined flange forces, equal to zero in uniform beams, and by normal stress gradients arising in the flanges. In consequence, circumferential shear stresses had linear components evoked by the axial force and parabolic ones caused by the moment and shear. Balduzzi et al. (2017) demonstrated several approaches to calculating cross sections stress distribution within tapered thin-walled I-beams. The method proposed by the authors was distinguished by the difference between the calculated and actual values below $5 \%$ in all considered cases. This allowed one to develop a new generation of effective tools useful for design of such structural elements. Ghayesh (2018) presented a nonlinear vibration problems of axially functionally graded shear deformable tapered beams with constant depth and varying width along length. Magnucki (2019) analysed simply supported sandwich beams and symmetrical I-beams. The cases of three-point bending and uniformly distributed load were considered. Various models of planar cross section deformation were taken into account. The principle of stationary total potential energy was used to derive differential equations of equilibrium that enabled calculation of beam deflection with consideration of the shear effect. Magnucki et al. (2019) provided a comparative analysis of the stress state in bending of a tapered cantilever beam. The stresses computed analytically, based on literature sources, and numerically (FEM) were compared with each other showing good agreement of both result series. Bertolini et al. (2019) derived analytical expressions for six stress components in untwisted, straight, thin-walled beams with rectangular and circular cross sections, distinguished by constant taper and subjected to forces acting in the cross section. The taper not only modifies stress magnitudes and distributions but gives rise to stress components, zeroed in prismatic beams. It was shown that increased taper shortens fatigue life of the beam.

The aim of the work is to develop an analytical model of bending of an expanded-tapered beam based on the adopted original deformation theory of planar cross section. The beam deflection was then calculated with consideration of the shear effect and stress distribution based on this theory. The novelty of the present approach consists in assumption of an individual theory-hypothesis determining the pattern of beam cross section deformation. The hypothesis enables one to take into account the shear stress distribution and the shear effect arising while bending of the beam.

The subject of the paper is a simply supported symmetrically expanded-tapered beam of rectangular cross section under three-point bending (Fig. 1).

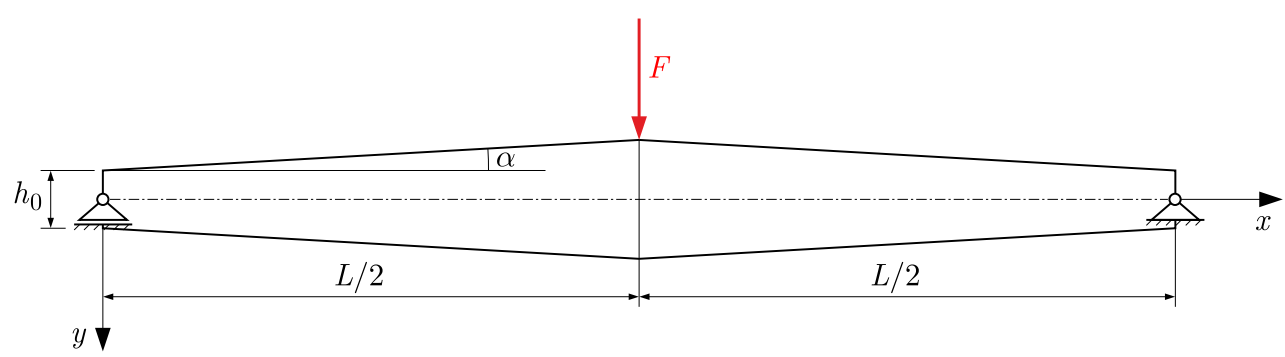

Fig. 1. Scheme of a simply supported expanded-tapered beam under three-point bending

The rectangular cross section is distinguished by constant width $b$ and linear variable depth

$$
h(\xi)=h_{0} \widetilde{h}(\xi)
$$

where: $h_{0}$ - depth of the beam ends, $\widetilde{h}(\xi)=1+2 \xi \tan \alpha$-dimensionless depth of the beam, $\alpha-$ taper angle, $\xi=x / h_{0}$ - dimensionless coordinate $(0 \leqslant \xi \leqslant \lambda / 2), L$-length, $\lambda=L / h_{0}$ - relative length of the beam. 


\section{Analytical model of the beam}

The nonlinear hypothesis of deformation of planar cross section after bending of the beam is assumed (Fig. 2).

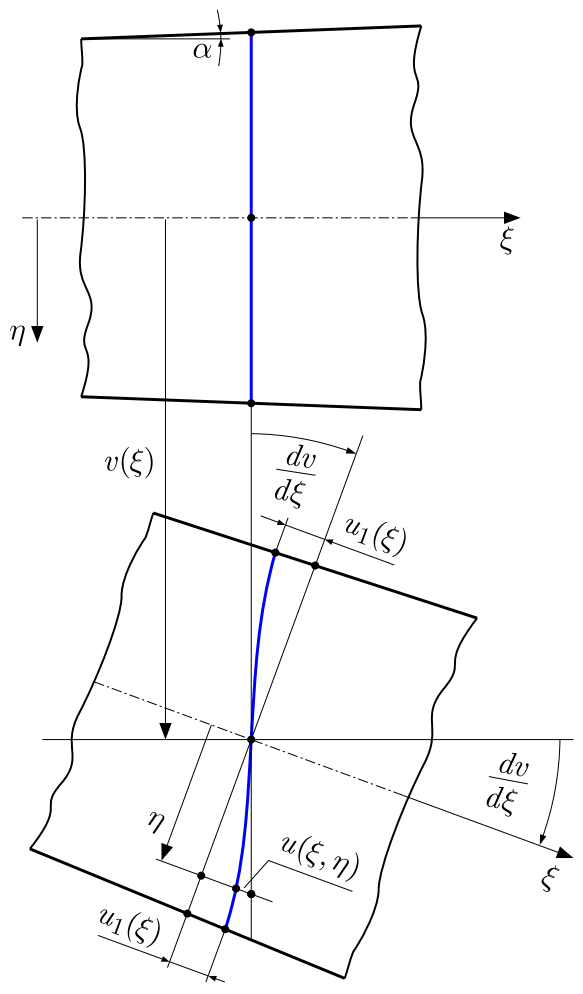

Fig. 2. Scheme of deformation of planar cross section after beam bending; $v(\xi)$ - deflection, $u_{1}(\xi)$ - displacement of points located at the upper/lower surfaces

The longitudinal displacement is of the following form

$$
u(\xi, \eta)=-\eta \frac{d v}{d \xi}+f_{d}(\xi, \eta) u_{1}(\xi)
$$

where $f_{d}$ is the original deformation function

$$
f_{d}(\xi, \eta)=\left[3-4(1-4 \xi \tan \alpha)\left(\frac{\eta}{\widetilde{h}(\xi)}\right)^{2}\right] \frac{\eta}{\widetilde{h}(\xi)^{2}}
$$

and $\eta=y / h_{0}$ - dimensionless coordinate. This function is a generalization of the nonlinear theory of beams with consideration of the shear effect.

Therefore, the strains take the following form

$$
\begin{aligned}
& \varepsilon_{x}(\xi, \eta)=\frac{\partial u}{h_{0} \partial \xi}=-\left[\eta \frac{d^{2} v}{d \xi^{2}}-f_{d}(\xi, \eta) \frac{d u_{1}}{d \xi}-\frac{\partial f_{d}}{\partial \xi} u_{1}(\xi)\right] \frac{1}{h_{0}} \\
& \gamma_{x, y}(\xi, \eta)=\frac{\partial u}{h_{0} \partial \eta}+\frac{d v}{h_{0} d \xi}=\frac{\partial f_{d}}{\partial \eta} u_{1}(\xi) \frac{1}{h_{0}}
\end{aligned}
$$

where derivatives of the deformation function are as follows

$$
\begin{aligned}
& \frac{\partial f_{d}}{\partial \xi}=-12\left[1-4(1-2 \xi \tan \alpha)\left(\frac{\eta}{\widetilde{h}(\xi)}\right)^{2}\right] \frac{\eta}{\widetilde{h}(\xi)^{3}} \tan \alpha \\
& \frac{\partial f_{d}}{\partial \eta}=3\left[1-4(1-4 \xi \tan \alpha)\left(\frac{\eta}{\widetilde{h}(\xi)}\right)^{2}\right] \frac{\eta}{\widetilde{h}(\xi)^{2}}
\end{aligned}
$$


The stresses according to Hooke's law

$$
\sigma_{x}(\xi, \eta)=E \varepsilon_{x}(\xi, \eta) \quad \tau_{x y}(\xi, \eta)=\frac{E}{2(1+\nu)} \gamma_{x y}(\xi, \eta)
$$

where: $E$ - Young's modulus, $\nu$ - Poisson's ratio.

The bending moment

$$
M_{b}(\xi)=b h_{0}^{2} \int_{-\widetilde{h}(\xi) / 2}^{\widetilde{h}(\xi) / 2} \sigma_{x}(\xi, \eta) \eta d \eta
$$

Substituting expression $(2.4)_{1}$ for the normal stress, after integration with respect to depth of the beam, one obtains the following equation

$$
\frac{1}{12} \widetilde{h}(\xi)^{3} \frac{d^{2} v}{d \xi^{2}}-\frac{1}{5}(1+\xi \tan \alpha) \widetilde{h}(\xi) \frac{d u_{1}}{d \xi}+\frac{2}{5}(1+3 \xi \tan \alpha) u_{1}(\xi) \tan \alpha=-\frac{F}{2 E b} \xi
$$

where the bending moment $M_{b}(\xi)=F \xi h_{0} / 2$ is taken into account.

The shear force, with consideration of shear stress $(2.4)_{2}$, after integration with respect to depth of the beam takes the following form

$$
T(\xi)=b h_{0} \int_{-\widetilde{h}(\xi) / 2}^{\widetilde{h}(\xi) / 2} \tau_{x y}(\xi, \eta) d \eta=\frac{E b}{1+\nu} u_{1}(\xi)
$$

The classical analytical description of the three-point bending of a beam of length $L$ is as follows

$$
T(x)=\left\{\begin{array}{lll}
\frac{F}{2} & \text { for } & 0 \leqslant x<\frac{L}{2} \\
0 & \text { for } & x=\frac{L}{2} \\
-\frac{F}{2} & \text { for } & \frac{L}{2}<x \leqslant L
\end{array}\right.
$$

This shear force function is discontinuous for $x=L / 2$, moreover its derivative is equal to zero. Consequently, according to expression (2.7), the displacement function $u_{1}(\xi)$ is also discontinuous for $x=L / 2$, and its derivative is zero. Therefore, equation (2.6) becomes incomplete due to zeroing of the derivate, $\left(d u_{1} / d x=0\right)$.

Hence, with a view to ensure continuity of the shear force and displacement functions, the shear force function for the three-point bending is assumed in the following form

$$
T(\xi)=\frac{1}{2} \tanh \left(\frac{\lambda}{2}-\xi\right) F
$$

Therefore, based on above expressions (2.7) and (2.8), the function $u_{1}(\xi)$ - displacement (Fig. 2) takes the following form

$$
u_{1}(\xi)=\frac{1}{2}(1+\nu) \tanh \left(\frac{\lambda}{2}-\xi\right) \frac{F}{E b}
$$

Substituting this function into Eq. (2.6) and making simple transformation, one obtains the equation

$$
\frac{d^{2} v}{d \xi^{2}}=-6\left\{\frac{1+\nu}{5}\left[\frac{1+\xi \tan \alpha}{\cosh ^{2}(\lambda / 2-\xi)} \widetilde{h}(\xi)+2 \tanh \left(\frac{\lambda}{2}-\xi\right)(1+3 \xi \tan \alpha) \tan \alpha\right]+\xi\right\} \frac{1}{\widetilde{h}(\xi)^{3}} \frac{F}{E b}
$$


Consequently, after integration one obtains

$$
\frac{d v}{d \xi}=-6\left\{C_{1}+\frac{1}{5}(1+\nu)\left[J_{1}(\xi)+2 J_{2}(\xi)\right]-\frac{1}{8} \frac{1+4 \xi \tan \alpha}{[\widetilde{h}(\xi) \tan \alpha]^{2}}\right\} \frac{F}{E b}
$$

where $J_{1}$ and $J_{2}$ - numerically calculated integrals

$$
J_{1}=\int \frac{1+\xi \tan \alpha}{\cosh ^{2}(\lambda / 2-\xi)} \frac{1}{\widetilde{h}(\xi)^{2}} d \xi \quad J_{2}=\int \tanh \left(\frac{\lambda}{2}-\xi\right)(1+3 \xi \tan \alpha) \frac{\tan \alpha}{\widetilde{h}(\xi)^{3}} d \xi
$$

and $C_{1}$ - integration constant determined from the condition $d v /\left.d \xi\right|_{\lambda / 2}=0$

$$
C_{1}=\frac{1}{8} \frac{1+2 \lambda \tan \alpha}{[\widetilde{h}(\lambda / 2) \tan \alpha]^{2}}-\frac{1}{5}(1+\nu)\left(\left.J_{1}\right|_{0} ^{\lambda / 2}+\left.2 J_{2}\right|_{0} ^{\lambda / 2}\right)
$$

Therefore, integrating Eq. (2.11) and taking into account the boundary condition $v(0)=0$, simple transformation enables one to formulate the maximum deflection of the beam

$$
v_{\text {max }}^{(\text {Analyt })}=v\left(\frac{1}{2} \lambda\right)=\widetilde{v}_{\text {max }}^{(\text {Analyt })} \frac{F}{E b}
$$

where the dimensionless maximum deflection is

$$
\begin{aligned}
& \widetilde{v}_{\text {max }}^{\text {Analyt })}=6\left\{\frac{\ln \widetilde{h}(\lambda / 2)}{8 \tan ^{3} \alpha}-\frac{2+3 \lambda \tan \alpha}{16[\widetilde{h}(\lambda / 2) \tan \alpha]^{2}} \lambda\right. \\
& \left.\quad-\frac{1+\nu}{5}\left[\left(\left.J_{1}\right|_{0} ^{\lambda / 2}+\left.2 J_{1}\right|_{0} ^{\lambda / 2}\right) \lambda+\left.J_{21}\right|_{0} ^{\lambda / 2}+\left.2 J_{22}\right|_{0} ^{\lambda / 2}\right]\right\}
\end{aligned}
$$

and two numerically calculated integrals are

$$
\begin{aligned}
\left.J_{21}\right|_{0} ^{\lambda / 2} & =\int_{0}^{\lambda / 2} \int \frac{1+\xi \tan \alpha}{\cosh ^{2}(\lambda / 2-\xi)} \frac{1}{\widetilde{h}(\xi)^{2}} d \xi^{2} \\
\left.J_{22}\right|_{0} ^{\lambda / 2} & =\int_{0}^{\lambda / 2} \int \tanh \left(\frac{\lambda}{2}-\xi\right)(1+3 \xi \tan \alpha) \frac{\tan \alpha}{\widetilde{h}(\xi)^{3}} d \xi^{2}
\end{aligned}
$$

Shear stress $(2.4)_{2}$ with consideration of expressions $(2.3)_{2}$ and (2.9) is expressed as follows

$$
\tau_{x y}(\xi, \eta)=\widetilde{\tau}_{x y}(\xi, \eta) \frac{F}{b h_{0}}
$$

where the dimensionless shear stress is

$$
\widetilde{\tau}_{x y}(\xi, \eta)=\frac{3}{4}\left[1-4(1-4 \xi \tan \alpha)\left(\frac{\eta}{\widetilde{h}(\xi)}\right)^{2}\right] \frac{1}{\widetilde{h}(\xi)^{2}} \tanh \left(\frac{\lambda}{2}-\xi\right)
$$

Expressions (2.13) and (2.15) make a basis for detailed studies of bending of the beams.

\section{Numerical FEM model of the beam}

Numerical analysis of the expanded-tapered beams is carried out with the SolidWorks software package. Symmetry of the beam structure allows one to consider the model including a quarter of the whole beam (Fig. 3). The adopted boundary conditions simulate behaviour of the beam.

The vertical longitudinal middle plane of the beam coincides with the $x y$-plane of the coordinate system. The neutral axis of the beam is collinear with the $x$-axis, while the $y$-axis aims downward. The beam model is divided into 3D tetrahedral finite elements with 16 Jacobian points. An example of the mesh is shown in Fig. 4. 


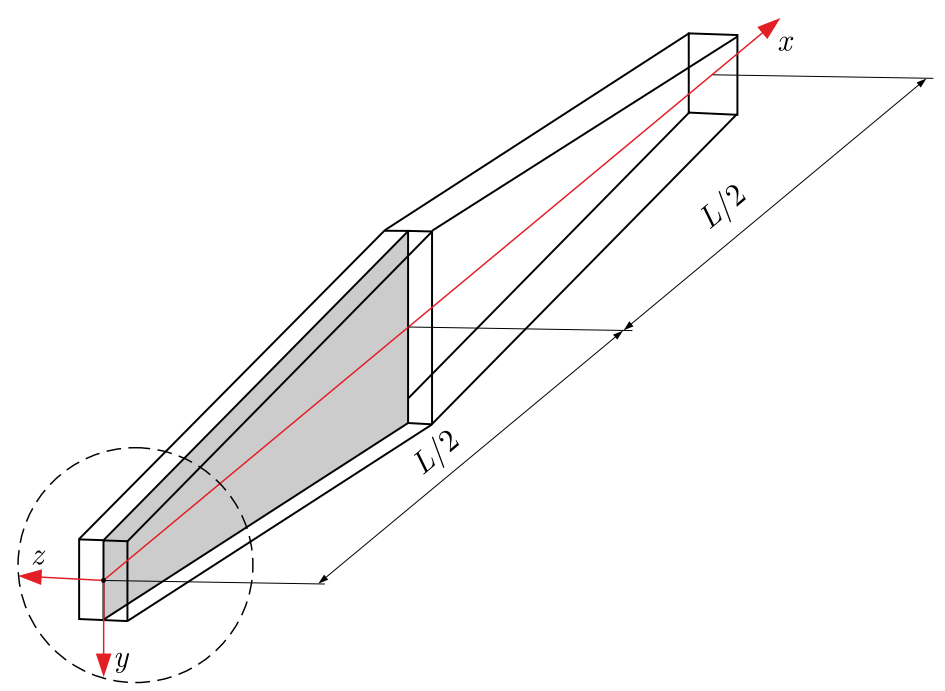

Fig. 3. Model of the expanded-tapered beam adopted for purposes of FEM computation

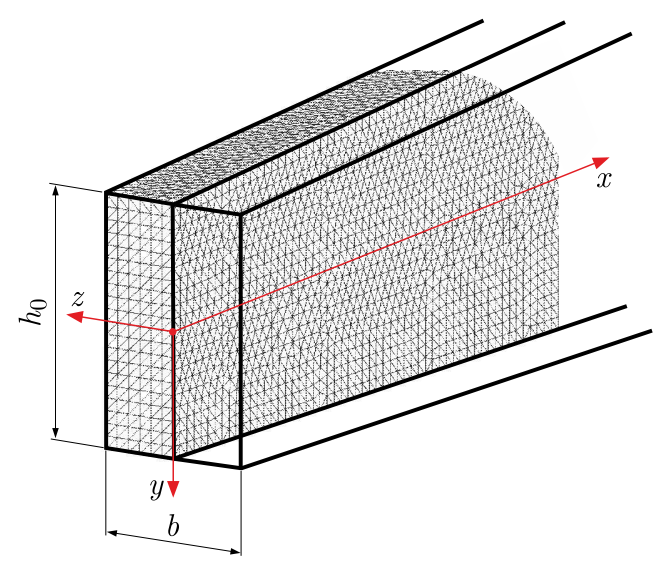

Fig. 4. A part of an exemplary FEM mesh (in the beam area marked approximately in Fig. 3 with the dotted circle)

The following boundary conditions corresponding to the case of a simply supported beam are assumed:

- for $x=0$ - a simple support at the beam, i.e. the $y$ displacements at the $y z$-plane are zero;

- for $x=L / 2$ at the middle of the beam - the $x$ displacements are zero;

- for $z=0$, i.e. at the vertical longitudinal middle plane of the beam - the $z$ displacements are zero.

\section{Analytical and FEM-numerical example studies}

The exemplary studies of expanded-tapered beams under three-point bending are carried out for the following data: initial depth $h_{0}=10 \mathrm{~mm}$, width $b=60 \mathrm{~mm}$ and length $L=4000 \mathrm{~mm}$, dimensionless length $\lambda=40$, taper angle $\alpha=0^{\circ}, 1^{\circ}, \ldots, 5^{\circ}$, Young's modulus $E=200 \mathrm{GPa}$, Poisson's ratio $\nu=0.3$.

The dimensionless maximum deflections of the beams are specified in Table 1.

The dimensionless shear stresses in the selected cross sections of the example beams calculated analytically (2.15) and numerically FEM are specified in Tables 2, 3 and 4.

Taking into account deformation function (2.2) it may be noticed that for $\xi=1 / 4 \tan \alpha$, the function is linear (Fig. 6) and, in consequence, the shear stress is constant (equal to 0.333) in the 
Table 1. Dimensionless maximum deflections of the beams: analytical (2.13) and FEM

\begin{tabular}{|c|c|c|c|c|c|c|}
\hline$\alpha\left[^{\circ}\right]$ & 0 & 1 & 2 & 3 & 4 & 5 \\
\hline \hline$\widetilde{v}_{\text {max }}^{\text {Analyt })}$ & 16030.1 & 4802.2 & 2157.4 & 1181.8 & 729.0 & 486.9 \\
\hline$\widetilde{v}_{\max }^{(\text {FEM }}$ & 16032.0 & 4803.0 & 2157.0 & 1181.1 & 728.1 & 486.0 \\
\hline
\end{tabular}

Table 2. Dimensionless shear stresses: analytical (2.15) and FEM for $\zeta=0$

\begin{tabular}{|c|c|c|c|c|c|}
\hline$\alpha\left[^{\circ}\right]$ & 1 & 2 & 3 & 4 & 5 \\
\hline \hline$\widetilde{\tau}_{x y}(\eta=0)$ (analyt.) & 0.750 & 0.750 & 0.750 & 0.750 & 0.750 \\
\hline$\widetilde{\tau}_{x y}(\eta= \pm \widetilde{h}(\xi) / 2)($ analyt. $)$ & 0 & 0 & 0 & 0 & 0 \\
\hline$\widetilde{\tau}_{x y}(\eta=0)($ FEM $)$ & 0.723 & 0.715 & 0.706 & 0.698 & 0.691 \\
\hline$\widetilde{\tau}_{x y}(\eta= \pm h(\xi) / 2)($ FEM $)$ & 0.010 & 0.016 & 0.021 & 0.027 & 0.033 \\
\hline
\end{tabular}

Table 3. Dimensionless shear stresses: analytical (2.15) and FEM for $\zeta=1 /(4 \tan \alpha)$

\begin{tabular}{|c|c|c|c|c|c|}
\hline$\alpha\left[^{\circ}\right]$ & 1 & 2 & 3 & 4 & 5 \\
\hline \hline$\widetilde{\tau}_{x y}(\eta=0)$ (analyt.) & 0.333 & 0.333 & 0.333 & 0.333 & 0.333 \\
\hline$\widetilde{\tau}_{x y}(\eta= \pm \widetilde{h}(\xi) / 2)$ (analyt.) & 0.333 & 0.333 & 0.333 & 0.333 & 0.333 \\
\hline$\widetilde{\tau}_{x y}(\eta=0)($ FEM $)$ & 0.334 & 0.333 & 0.334 & 0.334 & 0.334 \\
\hline$\widetilde{\tau}_{x y}(\eta= \pm \widetilde{h}(\xi) / 2)($ FEM $)$ & 0.334 & 0.333 & 0.334 & 0.335 & 0.335 \\
\hline
\end{tabular}

Table 4. Dimensionless shear stresses: analytical (2.15) and FEM for $\zeta=15$

\begin{tabular}{|c|c|c|c|c|c|}
\hline$\alpha\left[^{\circ}\right]$ & 1 & 2 & 3 & 4 & 5 \\
\hline \hline$\widetilde{\tau}_{x y}(\eta=0)($ analyt. $)$ & 0.323 & 0.179 & 0.113 & 0.0781 & 0.0571 \\
\hline$\widetilde{\tau}_{x y}(\eta= \pm \widetilde{h}(\xi) / 2)$ (analyt.) & 0.338 & 0.375 & 0.356 & 0.328 & 0.300 \\
\hline$\widetilde{\tau}_{x y}(\eta=0)($ FEM $)$ & 0.323 & 0.180 & 0.115 & 0.079 & 0.052 \\
\hline$\widetilde{\tau}_{x y}(\eta= \pm \widetilde{h}(\xi) / 2)($ FEM $)$ & 0.338 & 0.375 & 0.356 & 0.327 & 0.299 \\
\hline
\end{tabular}

whole cross section. Moreover, for $\xi \leqslant 1 / 4 \tan \alpha$, the maximum value of the shear stress occurs at $\eta=0$ (Fig. 5). The opposite situation exists for $1 / 4 \tan \alpha \leqslant \xi$ when the maximum value of the shear stress occurs for $\eta= \pm \widetilde{h}(\xi) 2$ (Fig. 7).

Selected forms of deformation function (2.2) and dimensionless shear stress distributions (2.15) for the taper angle $\alpha=2^{\circ}$ and the dimensionless coordinates $\zeta=0, \xi=1 /(4 \tan \alpha)$, $\zeta=15$ are shown in Figs. 5-7.

Comparison of the above results with the values obtained analytically and specified in Tables 1 to 4 and depicted in Figs. 5 to 8, respectively, indicates very good convergence of both series of the results. A little worse concurrence of the results is observed in the case of the shear stresses for $\zeta=0$. It is due to the fact that the $y$ displacements of the whole wall coinciding with the $y z$-plane are zero, that is not exactly equivalent to the simple support adopted in the analytical approach.

Comparison of the values of maximum deflection of the beam calculated analytically and FEM-numerically is graphically presented in Fig. 9.

\section{Conclusions}

Beams of varying cross section dimensions are widely used in many fields of structural mechanics. Among such structures, extended-tapered beams may be distinguished. In order to precisely study phenomena arising while their bending, the shear stress should not be ignored. 
(a)

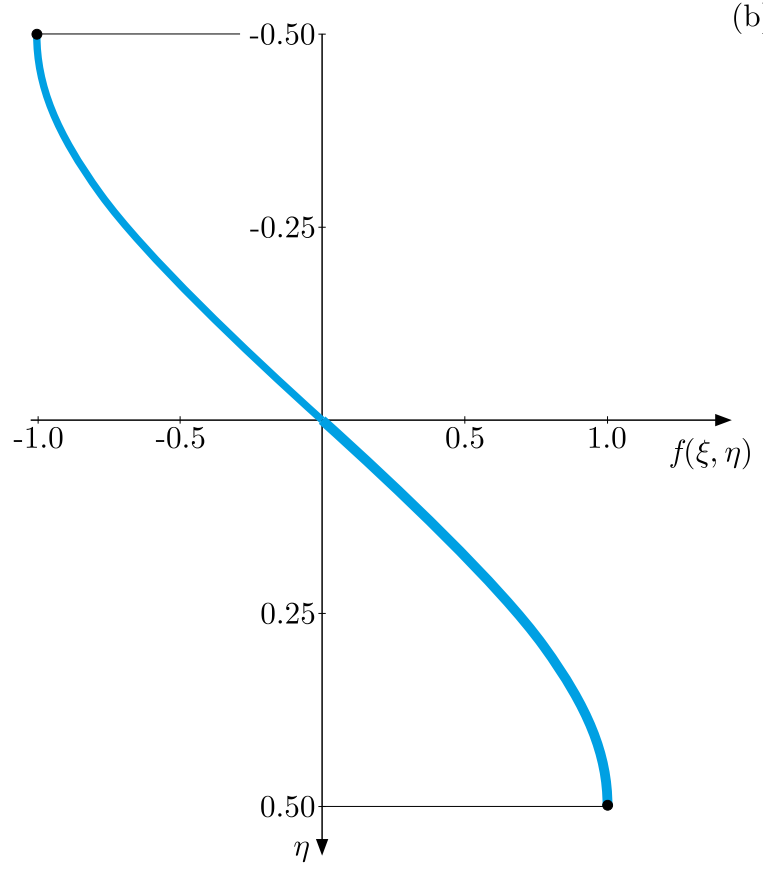

(b)

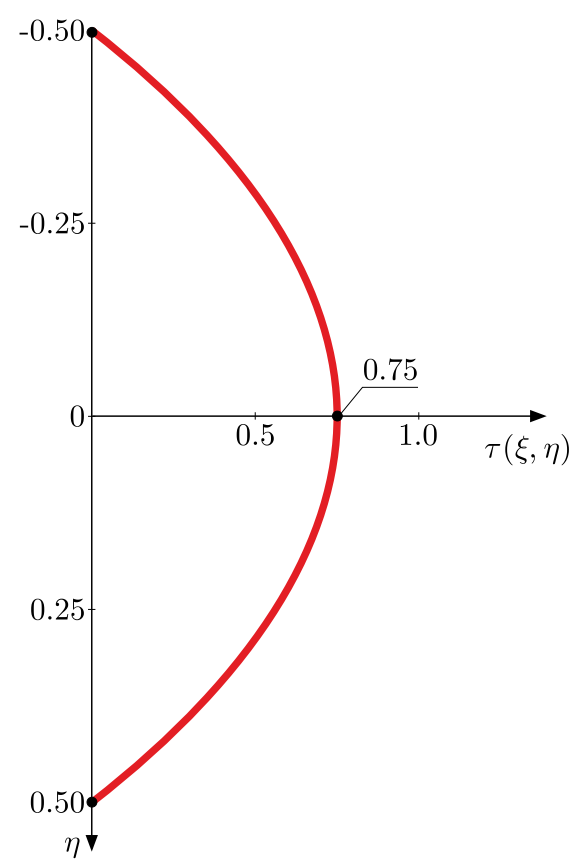

Fig. 5. Shape of analytically calculated deformation function (2.2): (a) diagram of the shear stress $(2.15)$, (b) for $\zeta=0$

(a)

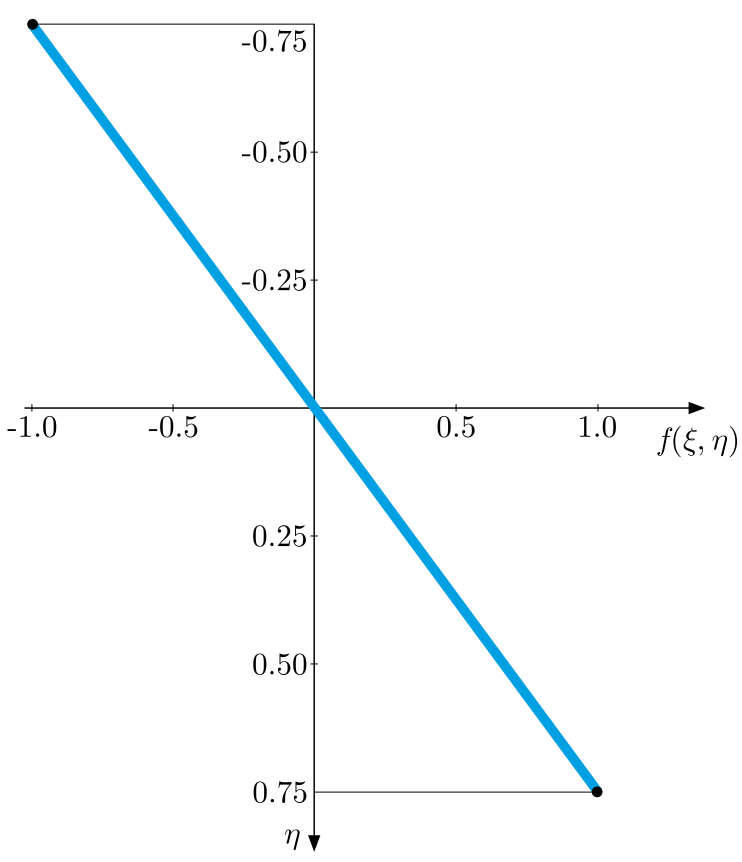

(b)

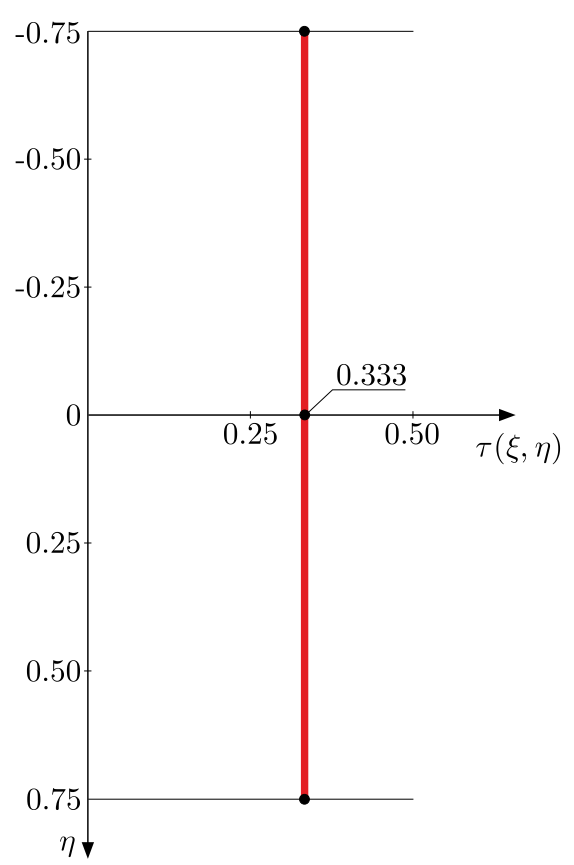

Fig. 6. Shape of analytically calculated deformation function (2.2): (a) diagram of the shear stress $(2.15),(\mathrm{b})$ for $\xi=1 /(4 \tan \alpha)$

Consideration of the shear effect on the behaviour of the bent expanded-tapered beam requires assumption of a hypothesis of deplanation of its initially planar cross section. Such an original, non-linear hypothesis is adopted in the present paper. It is formulated with the use of a polynomial function. The hypothesis enables one to determine location of the singular cross section which remains planar while bending (Fig. 6a, for $\xi=1 /(4 \tan \alpha)$. 
(a)

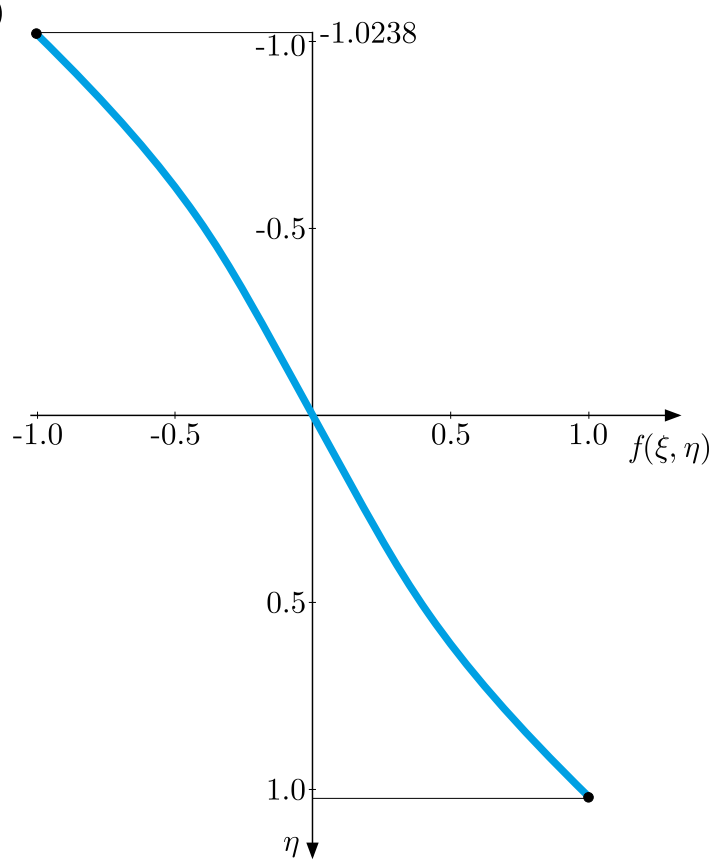

(b)

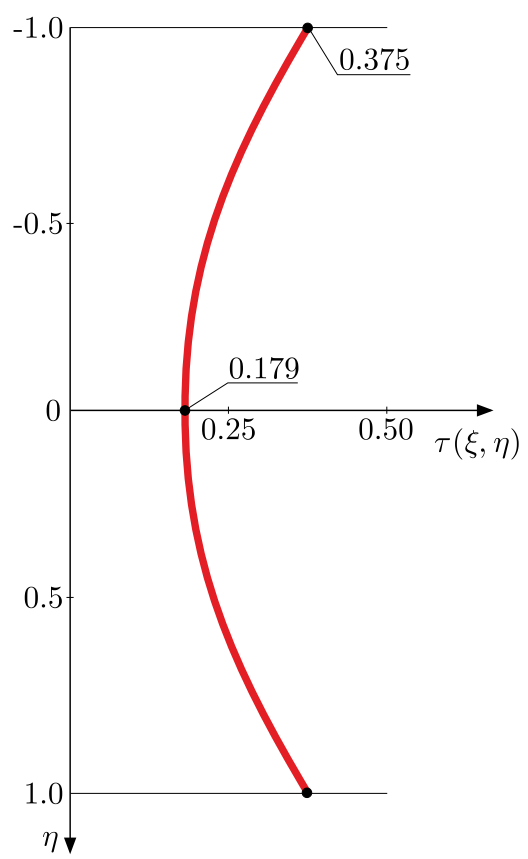

Fig. 7. Shape of analytically calculated deformation function (2.2): (a) diagram of the shear stress (2.15), (b) for $\zeta=15$

(a)

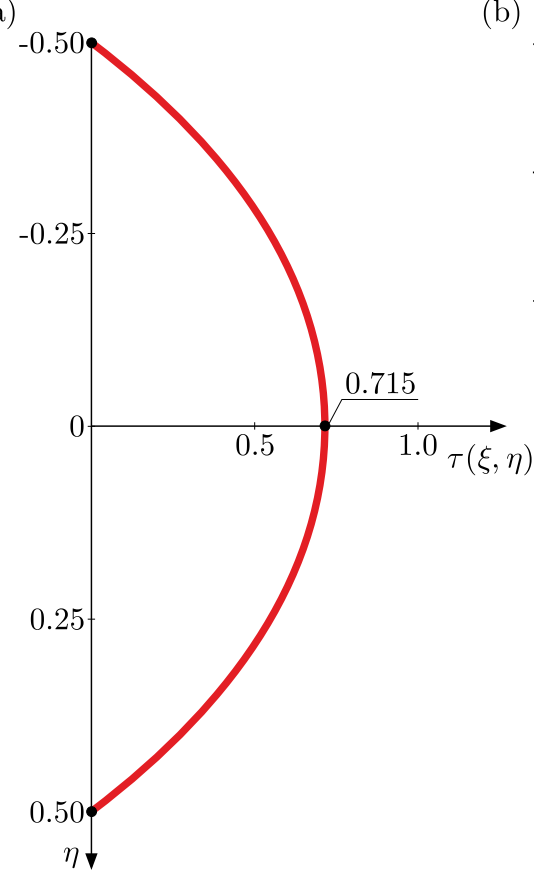

(b)

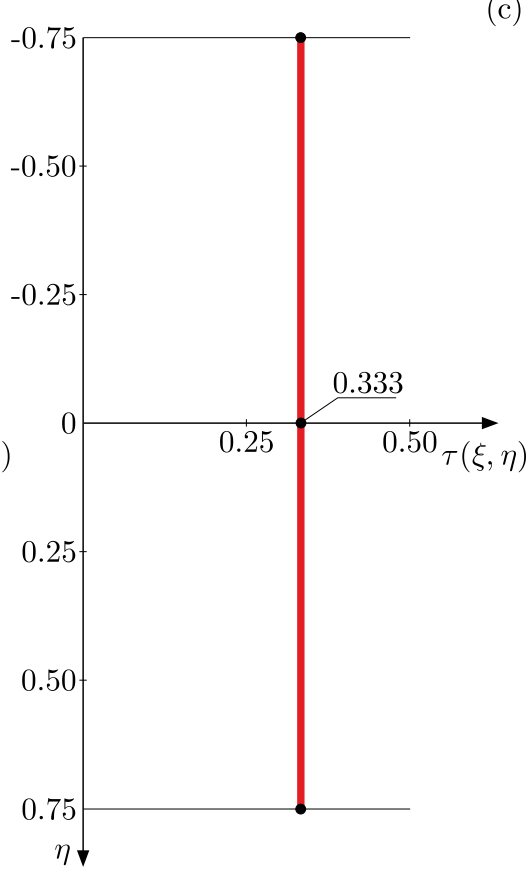

(c)

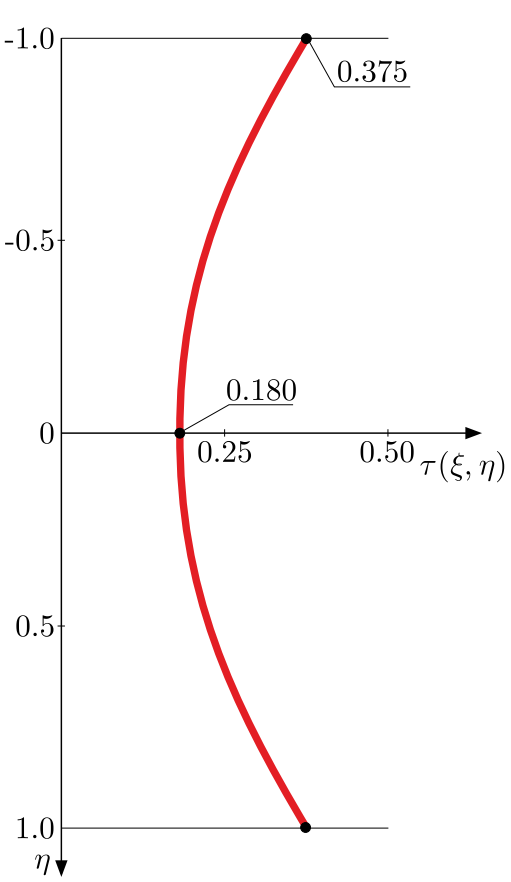

Fig. 8. Diagrams of the shear stress calculated with FEM: (a) for $\zeta=0$, (b) for $\xi=1 /(4 \tan \alpha)$,

(c) for $\zeta=15$

The analytical calculation results achieved with the help of the hypothesis, namely the deflection and shear stress distribution in several locations of the beam, have been compared to the ones obtained numerically with the Finite Element Method.

The comparison demonstrated good effectiveness of the approach. Thereby, the proposed hypothesis of deplanation may be considered as a promising method of bending analysis of expanded-tapered beams.

In order to ensure continuity of the shear force and displacement, assumed shear force function (2.8) for exemplary beams of relative length $\lambda=40$ takes the form shown in Fig. 10. 


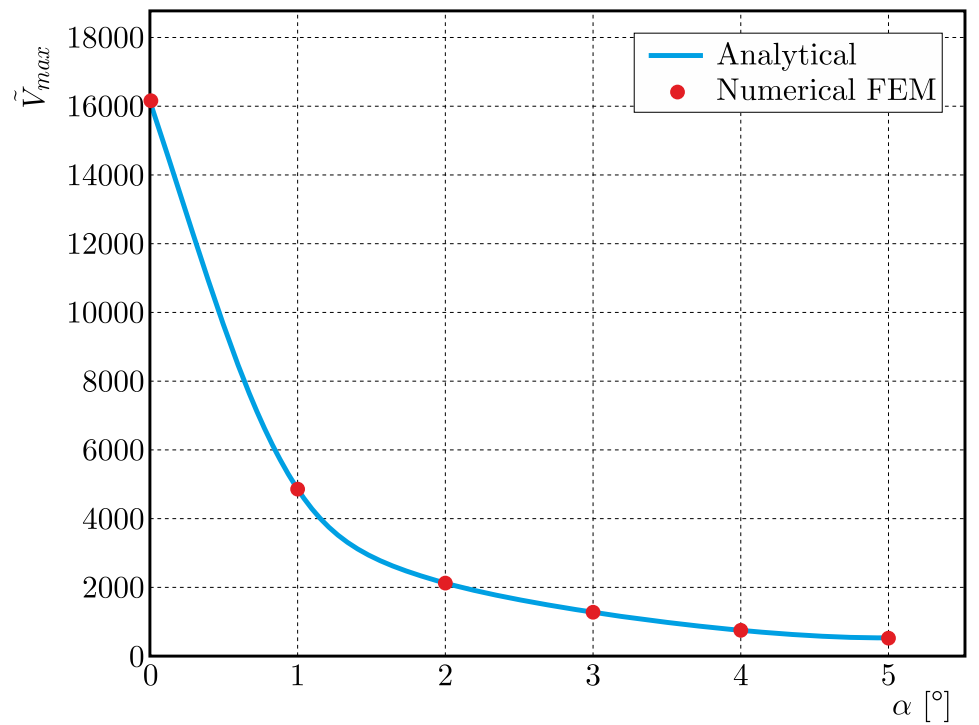

Fig. 9. Maximum deflection of the beam obtained analytically and numerically

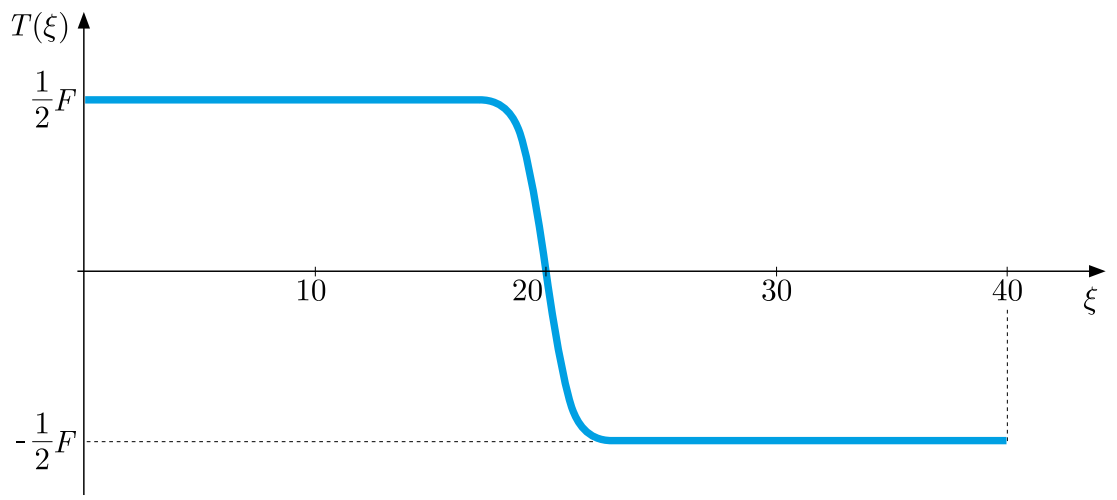

Fig. 10. Diagram of shear force function (2.8) for three-point bending of exemplary beams

This function assumed in the analytical model provides continuity of the longitudinal displacements $u_{1}(x)$ (Fig. 2). Such a function is meaningful for practical application in the case of a concentrated force acting on the beam.

\section{References}

1. Attarnejad R., Shahba A., Semnani S.J., 2011, Analysis of non-prismatic Timoshenko beams using basic displacement functions, Advanced Structural Engineering, 14, 2, 319-332

2. Auciello N.M., Ercolano A., 2004, A general solution for dynamic response of axially loaded non-uniform Timoshenko beams, International Journal of Solids and Structures, 41, 18-19, 4861$-4874$

3. Auricchio F., Balduzzi G., Lovadina C., 2015, The dimensional reduction approach for 2D non-prismatic beam modelling: A solution based on Hellinger-Reissner principle, International Journal of Solids and Structures, 63, 264-276

4. Balduzzi G., Aminbaghai M., Sacco E., Füssl J., Eberhardsteiner J., Auricchio F., 2016, Non-prismatic beams: A simple and effective Timoshenko-like model, International Journal of Solids and Structures, 90, 236-250

5. Balduzzi G., Hochreiner G., Füssl J., 2017, Stress recovery from one dimensional models for tapered bi-symmetric thin-walled I beams: deficiencies in modern engineering tools and procedures, Thin-Walled Structures, 119, 934-944 
6. Bertolini P., Eder M.A., Taglialegne L., Valvo P.S., 2019, Stresses in constant tapered beams with thin-walled rectangular and circular cross sections, Thin-Walled Structures, 137, 527-540

7. Carrera E., Giunta G., 2010, Refined beam theories based on a unified formulation, International Journal of Applied Mechanics, 2, 1, 117-143

8. Dado M., Al-Sadder S., 2005, A new technique for large deflection analysis of non-prismatic cantilever beams, Mechanics Research Communications, 32, 6, 692-703

9. De Rosa M.A., Lippiello M., 2009, Natural vibration frequencies of tapered beams, Engineering Transactions, 57, 1, 45-66

10. Ghayesh M.H., 2018, Nonlinear vibration analysis of axially functionally graded shear-deformable tapered beams, Applied Mathematical Modelling, 59, 583-596

11. Huang Y., Wu J.X., Li X.F., Yang L.E., 2013, Higher-order theory for bending and vibration of beams with circular cross section, Journal of Engineering Mathematics, 80, 1, 91-104

12. MAalEK S., 2004, Shear deflections of tapered Timoshenko beams, International Journal of Mechanical Sciences, 46, 5, 783-805

13. MagnuCKi K., 2019, Bending of symmetrically sandwich beams and I-beams - Analytical study, International Journal of Mechanical Sciences, 150, 411-419

14. Magnucki K., Lewinski J., Stawecka H., 2019, Stress state in the tapered beam bending Analytical and numerical FEM studies, Rail Vehicles, 2, 1-8

15. RAJASEKARAN S., 2008, Buckling of fully and partially embedded non-prismatic columns using differential quadrature and differential transformation methods, Structural Engineering Mechanics, 28, 2, 221-238

16. RAJASEKARAN S., 2013, Free vibration of centrifugally stiffened axially functionally graded tapered Timoshenko beams using differential transformation and quadrature methods, Applied Mathematical Modelling, 37, 6, 4440-4463

17. Reddy J.N., 2010, Nonlocal nonlinear formulations for bending of classical and shear deformation theories of beams and plates, International Journal of Engineering Sciences, 48, 1507-1518

18. Shahba A., Attarnejad R., Tavanaie Marvi M., Hajilar S., 2011, Free vibration and stability analysis of axially functionally graded tapered Timoshenko beams with classical and non-classical boundary conditions, Composites Part B: Engineering, 42, 4, 801-808

19. Slivker V., 2007, Mechanics of Structural Elements: Theory and Applications, Springer, Berlin, Heidelberg, New York

20. Taha M.H., NAssar M., 2014, Analysis of axially loaded tapered beams with general end restraints on two-parameter foundation, Journal of Theoretical and Applied Mechanics, 55, 1, 215-225

21. Timoshenko S.P., 1921, On the correction for shear of the differential equation for transverse vibrations of prismatic bars, The London, Edinburgh, and Dublin Philosophical Magazine and Journal of Science, 41, 245, 744-746

22. Trahair N., Ansourian P., 2016, In-plane behaviour of web-tapered beams, Engineering Structures, 108, 47-52

23. WANG C.Y., 2012, Large post-buckling of heavy tapered elastica cantilevers and its asymptotic analysis. Brief Note, Archives of Mechanics, 64, 2, 207-220

24. Wang C.M., Reddy J.N., Lee K.H., 2000, Shear Deformable Beams and Plates, Elsevier, Amsterdam, Lausanne, New York, Shannon, Singapore, Tokyo

25. Zhou D., Cheung K., 2001, Vibrations of tapered Timoshenko beams in terms of static Timoshenko beam functions, ASME: Journal of Applied Mechanics, 68, 4, 596-602 